\title{
Extremely Red Galaxies in Abell 1835
}

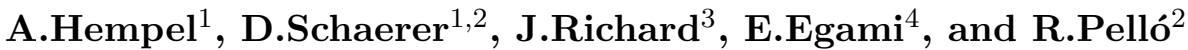 \\ ${ }^{1}$ Geneva Observatory, Ch. des Maillettes 51, CH-1290 Sauverny, Switzerland \\ email:Angela.Hempel@obs.unige.ch \\ ${ }^{2}$ Observatoire Midi-Pyréneés, 14 Avenue E. Belin, F-31400 Toulouse, France \\ ${ }^{3}$ Caltech Astronomy, MC105-24, Pasadena, CA 91125, USA \\ ${ }^{4}$ Steward Observatory, University Arizona, AZ 85721, USA
}

\begin{abstract}
We use multi-colour data of the gravitational lensing cluster Abell 1835 to search for high-z candidates (Richard et al. 2006) and extremely red galaxies.

Applying a colour criteria of R-K $\geqslant 5.6$ we have selected 12 EROs, of which 10(9) have no $\mathrm{R}(\mathrm{I})$-band detection. Two of these sources posses multiple detections in the ACS $\mathrm{z}_{850}$ band, possibly gravitationally bound objects.

Using the method of Pozzetti and Mannucci (2000) to classify an ERO either as evolved elliptical or dusty starburst, we find that two thirds of all objects have colours assigned to evolved ellipticals. One of the remaining 4 sources is the known sub-mm source SMMJ14009+0252.

The spectral energy distributions of sources with no optical detection show strong similarities to the SED of the high-z object of Mobasher et al. (2005), i.e. a degeneracy between a low- and high-z solution. However, for objects we accept the low-z solution as the better fit.

A detailed analyses of the colour properties and spectral energy distribution will be published in two forthcoming papers (Schaerer et al. , Hempel et al.).
\end{abstract}

Keywords. galaxies, fundamental parameters

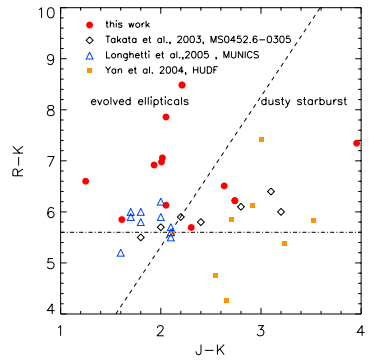

Figure 1. Colour-colour diagram of different EROs samples.
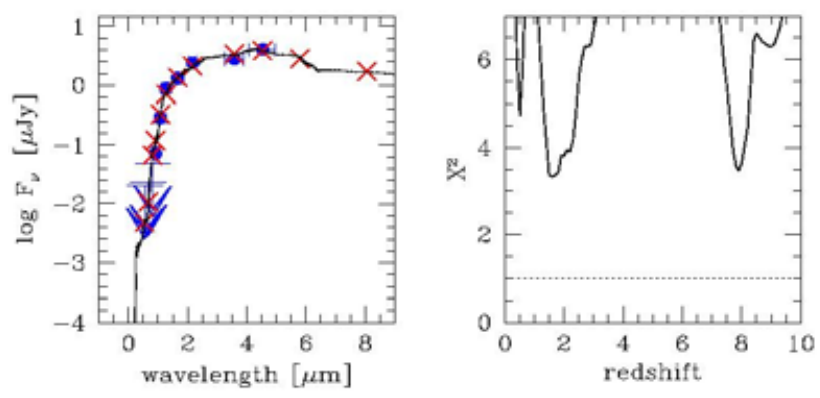

Figure 2. (left:) Best fit SED ( $F_{\nu}$ units in microJansky) for ERO 1, obtained with templates from BCCWW group; here an elliptical galaxy with no additional extinction. (right:) Reduced $\chi^{2}$ as a function of $z$.

\section{References}

Mobasher et al. 2005, ApJ 635, 832.

Pozzetti \& Mannucci 2000, MNRAS 317, L17.

Richard et al. 2006, A\&GA 456, 861. 